\title{
Smartphone Based Autism Social Alert System
}

\author{
M. Chuah, M. DiBlasio \\ CSE Department \\ Lehigh University \\ Bethlehem, PA, USA \\ $\{$ chuah, mid212\}@cse.lehigh.edu
}

\begin{abstract}
Autism currently affects 1 in every 88 American children impairing their social interactions, communications and daily living. Often, parents, educators, and researchers need to purchase expensive equipment to help autistic children cope with challenges in their daily living. In this paper, we present the Smartphone-Based Autism Social Alert (SASA) system which we design to help such children. The SASA system uses the inexpensive sensors embedded within smartphones to facilitate the study of the autistic children's behaviors by recording and analyzing data collected from such embedded sensors in smartphones carried by autistic children. Our system can automatically detect their stereotypical behaviors such that early interventions can be taken by caregivers or teachers. In addition, the system can correlate environmental sensor data streams, e.g. audio background, with the occurrence of stereotypical behaviors so as to identify potential environmental factors that may trigger such behaviors. We also include some preliminary classification results on the sensor data which we have collected from Androidbased phones using the WEKA J.48 classifier. Our preliminary results show that simple features extracted from accelerometer readings are sufficient to give high accuracy rates when training is performed on a per user per device basis. Our audio classifier which uses 12 MFCC coefficients, average zero crossing rate, and energy can give an accuracy of $78.6 \%$ when evaluated using audio traces collected for seven audio categories. Additional extensive experiments will be carried out in the near future at a nearby secondary school for autistic children.
\end{abstract}

Keywords-mobile phone sensing; healthcare;autism;social alert

\section{INTRODUCTION}

Smartphones today not only provide computing and communication capabilities but are also equipped with many embedded sensors e.g. GPS, accelerometer, compass, microphone, etc. These sensors enable new applications to be developed for several domains e.g. environmental monitoring, healthcare, transportation, and social networks. In the healthcare domain, several mobile applications have been developed recently to encourage people to live a healthy lifestyle. For example, several health-oriented games referred to as exergames $[1,2]$ have been developed. Exergames are applications where players must engage in physical activities to play. Studies have also been done to see if exergaming contributes to improving physical activity levels and health outcomes in children [3]. Other applications monitor behaviors so that people can determine if they have healthy lifestyles $[4,5]$.

Autism Spectrum Disorders [6] affects 1 in every 88 American children. Autistic children typically have impaired social interactions, communications and face some challenges in their daily lives. They typically exhibit stereotypical behaviors [14] which are defined as repetitive motor or vocal sequences

M. Chuah's effort is supported by National Science Foundation Grants 1016296, 1217379 that may appear invariant in form. Several stereotypical behaviors have been identified $[15,16]$ e.g. body-rocking, hand waving, foot tapping, etc. Such stereotypical behaviors if unregulated, can become the dominant behavior of that individual with ASD [13], and may potentially interfere with his acquisition of new skills and performance of existing learnt skills [17]. Furthermore, it may interfere with their social integration in school and community settings. In addition, such behaviors may sometimes cause danger to the autistic children as well as to others e.g. their caregivers [18].

One reason why such behaviors are not studied thoroughly in the past is because appropriate tools for measuring the behavior are either not easily available or expensive to deploy. Most of the time, behavioral monitoring is based on caregivers' observations in classrooms or at home. The embedded sensors within smartphone allow human behaviors to be monitored. Such monitoring creates new opportunities to improve scientific understanding of correlations between autistic children's behaviors and their contexts, and possibly their health conditions.

In this paper, we present a preliminary prototype of a smartphone-based autism social alert system which we design to help autism children and their caregivers to cope with the challenges they face in their daily living. In particular, our system collects multiple sensor data streams from smartphones or wearable sensors and sends such data streams to a remote server. The sensor data streams can be accelerometer readings that can be used to predict a child's behavioral action or audio recordings that reflect the environmental context that child is in. Caregivers, e.g. teachers, or parents, are granted access to the remote server to annotate such collected data. The annotated data is used to train classifiers. One classifier is trained for recognizing different stereotypical behaviors such as hand-waving, foot tapping, etc, while another is trained to recognize environmental contexts e.g. classroom A, home, restaurant B etc. Later, newly collected data stream can be fed to our system, and the trained classifier can determine if a monitored individual has exhibited any stereotypical behaviors. It can also predict the location he is at based on the environmental sensor data readings. Upon detecting stereotypical behaviors, our system consults a knowledgebased database to determine what appropriate intervention steps should be taken e.g. sending an alert message to caregivers or playing an audio clip with familiar caregivers' voice to calm that individual. Furthermore, since our system can correlate environmental contexts with observed stereotypical behaviors, we hope to learn whether a particular 
environmental factor, e.g. a toilet flush sound, can trigger any stereotypical behavior of an autistic child. Last but not least, we present preliminary classification results obtained using our prototype for identifying certain stereotypical behaviors and audio clips using J.48 classifiers. Our trained classifier can distinguish stereotypical behaviors like foot tapping, jumping, hand waving, walking, sitting with an accuracy of $85 \%$ while our audio classifier can distinguish 7 audio categories with an accuracy of $78.6 \%$. This accuracy is higher than what can be achieved using the approach described in [25].

The rest of the paper is organized as follows: In Section 2, we summarize some existing work that we are aware of. In Section 3, we describe our autism social alert system. In Section 4, we describe the features we extract from the accelerometer readings for behavioral classifications, and present some preliminary classification results we obtained. In Section 5, we describe how we analyze the collected audio recordings using Short-Term Fourier Transform, Melfrequency cepstrum-based analysis to identify the environmental contexts of a particular monitored individual. In Section 6, we describe the preliminary prototype we have developed. In Section 7, we present some preliminary results we have obtained. In Section 8 , we conclude by discussing some near future work that we plan to do.

\section{RELATED WORK}

There are currently no tools for clinicians or caregivers to easily, reliably and accurately monitor autism children's stereotypical behaviors. Traditional measures rely primarily on paper-and-pencil recordings of teachers and parents based on direct observations or video-based methods [7].

Paper and pencil recordings typical involve recordings of the frequency and/or severity of stereotypical behaviors. Such recordings can have questionable accuracy and may not capture inter-individual variations in the amount and duration of stereotypical behaviors [8]. Direct observation measures are not reliable because (a) it is sometimes not possible to note environmental factors and record stereotypical behaviors simultaneously, (b) it is difficult to determine when an action has started and ended, (c) the stereotypical actions happen intermittently and it is not feasible for parents or caregivers to monitor that autistic child 24/7.

Video-based methods involve video recording of behaviors and annotation of such stereotypical actions by an expert offline. The ability to replay such videos makes such methods more reliable than paper-and-pencil methods. However, such methods are expensive, tedious and time consuming. Combining such recording with learning and semi-automatic tagging is an on-going research topic $[9,13]$.

Westeyn et al used pattern recognition algorithms to analyze accelerometer readings for detecting stereotypical behaviors [10]. $69 \%$ of continuous recognition tests were accurately detected in [10] using Hidden Markov Models but the data were acquired from individuals mimicking the actual behaviors rather than collected from children with ASD. Only 2-axis accelerometers are used and each user has to wear 3 sets of sensors, one on right wrist, one at the back of the waist and one at the left ankle. Furthermore, there is no collection of any sensor data that describes autistic children's environmental contexts.

Researchers have also demonstrated that wearable accelerometers can be used to detect human activities such as household activities, postures, exercises, etc [11,12,22]. In this work, we are focusing more on the issues that one will face when designing a system that can automatically recognize stereotypical behaviors in natural settings of autistic children such that it can be deployed in a real secondary school for autistic children.

The work done in [13] is the most relevant one to our work. The authors in [13] collect three sets of 3-axis accelerometer readings from 6 students who suffer ASDs. They use video camera to capture videos of the experiments such that such video captures could be synchronized with the accelerometer readings and used for annotation of activity. They also used J48 classifier to identify stereotypical motor movements. However, they do not collect any sensor data related to environmental contexts. In our project, we would like to correlate environmental contexts with the occurrence of stereotypical behaviors so that we can identify any environmental factor, e.g., certain type of sound, which may trigger stereotypical behaviors or emotional upsets. Once such a factor can be identified, intervention steps can be taken to prevent such behaviors.

\section{SMARTPHONE-BASEd AUTISM SOCIAL AlERT SYSTEM}

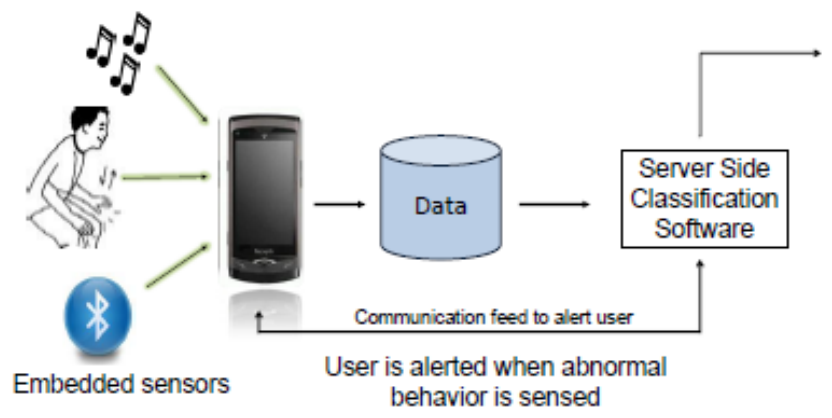

Fig 1 Smartphone-Based Autism Social Alert System

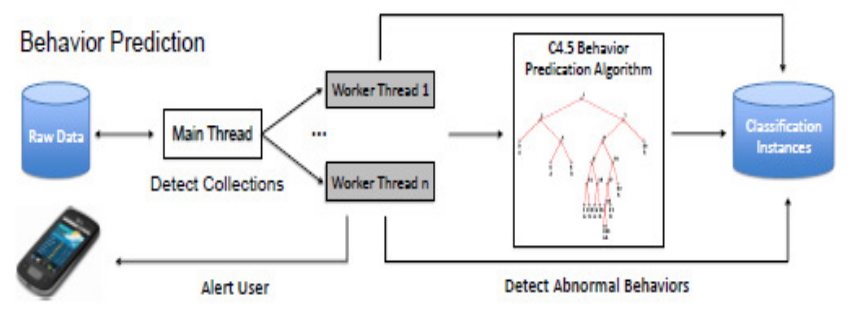

Fig 2 Server Side Software 
Our SASA system consists of Android-based smartphones or wearable sensors carried/worn by autism children, and a remote server as shown in Fig 1. Client software that runs on smartphones or wearable sensors collects and records continuous sensor data streams, e.g. GPS, Bluetooth, accelerometer and audio readings, and sends such data streams to a remote server. Some sensor data streams, e.g. the GPS, Bluetooth contact traces, and audio readings, provide information related to autistic children's environments while some sensor data streams are related to their behaviors e.g. accelerometer readings. The collected sensor data streams are sent from the monitoring devices to a remote server via $\mathrm{WiFi}$ connections. Our program can run either in "training" or "realtime" mode. When set in training mode, the sensor data stream can be stored in a file within the SD card, and uploaded to the remote server when a $\mathrm{WiFi}$ connection is available. When set in real-time mode, the sensor data stream will be sent continuously (currently set to every second but can be configurable in future) to a remote server and hence it expects continuous access to WiFi connectivity.

Caregivers are given accounts in that server to annotate collected accelerometer readings with labels such as walking, standing, or repetitive stereotype behaviors, e.g., bodyrocking, hand-waiving, jumping etc. They also annotate the environmental contexts associated with any environmental related sensor data streams e.g. audio recordings. The behavioral analysis and diagnostic program running in the remote server uses the labeled data to train a J.48 classifier for each monitored individual. To ensure that our server is scalable, we have different worker threads to process incoming sensor data streams as shown in Fig 2. WEKA tool is used to perform the J48 classification. There are two classifiers, one for recognizing stereotypical behaviors, and one for identifying environmental contexts e.g. classroom A, restaurant $\mathrm{B}$, home living room, bedroom etc. Our server program also learns to identify if certain environmental factors e.g. audio background trigger any stereotypical behaviors.

After training, the classifiers can then be used to predict if the new sensor data stream sent from a wearable device or smartphone contains data is similar to those observed when that individual is demonstrating certain stereotypical behaviors. If such a behavior is detected, the server consults a knowledge-based database to determine what interventions need to be taken e.g. generating an alert message to the caregiver or sending an audio clip with the voice of that individual's caregiver to help calm that individual. Furthermore, if an environmental factor has been known to trigger stereotypical behaviors, our autism social alert system will be able to send warning messages (in text or audio formats) to calm that individual such that any such behaviors can be prevented.

There are two key components in our system, namely (a) a trained classifier that can identify different stereotypical behaviors from accelerometer readings, (b) another classifier that can identify the environmental contexts of that monitored individual. We provide more details on these two components in Section IV and Section V.

\section{DATA Mining OF ACCELEROMETER READINGS}

In this section, we present some results we obtained by analyzing accelerometer readings collected from Androidbased smartphones when volunteered students perform different actions e.g. standing, walking, hand waving, jumping etc. We use the J48 classifier available in the WEKA package [19] for our data mining purppose. We first train the J48 classifier using a training dataset which consists of collected traces of accelerometer readings when various students perform different actions. During testing, the monitoring software running on an Android phone sends 4 samples of 3axis accelerometer readings every second to a remote server. The remote server forms a new test instance using a sliding window of 16 samples with an overlap of 4 samples. The server produces a classifier output for each test instance, and stores the result in our mysql database.

\section{A. Data Analysis of Different Behaviors}

Fig 3 plots the variances of the 3 -axis accelerometer readings for two behaviors, namely rapid hand movement (green) and jumping (blue). Fig 4(a) shows the variances in the 3-axis accelerometer readings for the rapid hand movement action while Fig 4(b) shows the variances in the 3-axis accelerometer readings for the jumping action. The blue line is the $\mathrm{X}$-axis reading, the red line is the $\mathrm{Y}$-axis reading+10 while the green line is the $\mathrm{Z}$-axis reading+10. One can see that different behaviors produce distinct sets of variances and hence such features can be used as training features to recognize different behaviors.

We have collected at least 20 traces per user for five different actions namely standing, walking, hand-waving, jumping, and foot tapping. We then compute variances of 16 samples of the 3-axis accelerometer readings, and use these as training features to classify different behaviors. Then, we use additional collected traces as test traces.

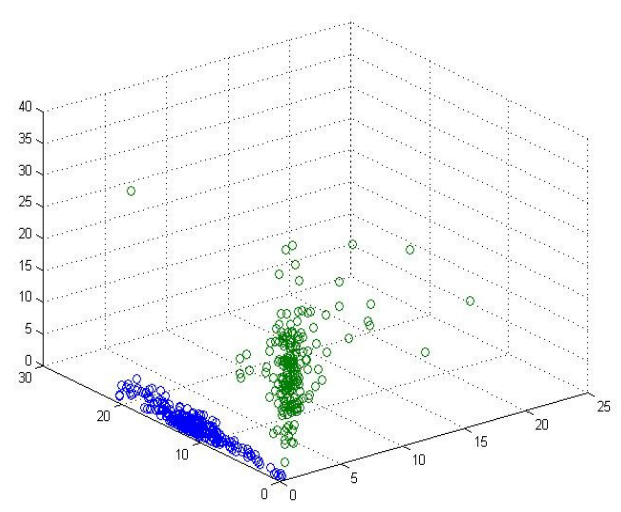

Fig 3 Plots of 3-axis accelerometer readings for different actions 


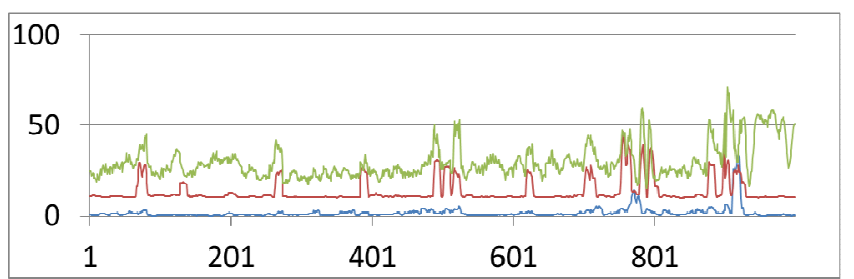

Fig 4(a) Variances in the 3-axis accelerometer readings for rapid hand movement.

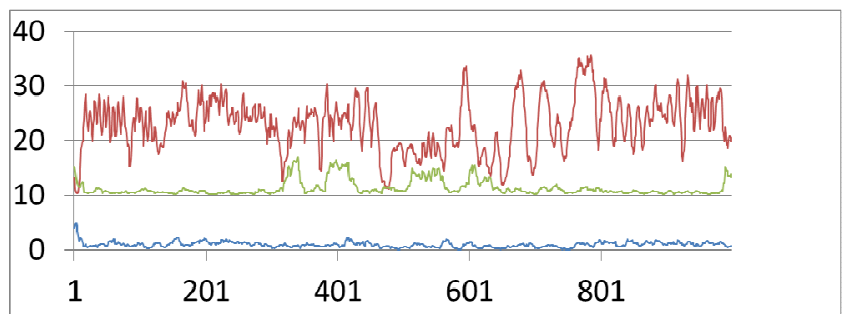

Fig 4(b) Variances in the 3-axis accelerometer readings for Jumping.

In our first set of experiments, the test traces we collect only consist of one action in each trace. We tried using both independent windows of 16 samples or sliding windows (also 16 samples) to predict the action using the trained J48 classifier. We obtained $91 \%$ accuracy rate using independent window and $89 \%$ accuracy using sliding window.

In our second set of experiments, we use test traces that consist of several behaviors. Fig 5 shows the classifier output of one particular test trace. The blue line shows the predicted behaviors while the red line shows the actual behaviors. As one can see, sometimes that user moves while he is sitting and hence the classifier will output "hand waving" (the phone is in that user's pocket when the accelerometer readings are collected). Similarly, a user may occasionally stop while performing the foot tapping action, and hence the classifier may conclude that the user is sitting. One way to remove such errors is to let our software conclude that a certain behavior is being performed only if the classifier outputs $M=5$ consecutive answers that a particular action is being performed especially when there is a change of behaviors e.g. from sitting to foot tapping.

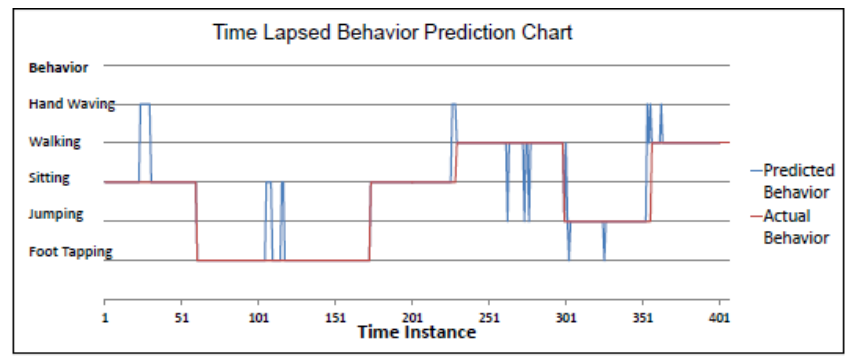

Fig 5 Classifier Output Fed with A trace with multiple actions

\section{DAta Mining OF AUdio BACKground}

One of the environmental factors we monitor is the audio background. Some stereotypical behaviors are triggered in the presence of some audio sounds that the autistic children dislike. For example, one autistic kid is known to show emotional distress whenever he hears toilet flush sound at unfamiliar places. Thus, we want to build a classifier that can recognize different environments using recorded audio clips such that appropriate intervention steps can be taken to prevent stereotypical behaviors once we identify the triggering environmental factors.

We first collect different types of sounds e.g. toilet flushing, telephone ringing and background noise of different empty rooms. Then, we analyze such audio recordings using the short-time fourier transform (STPFT) [21]. STPFT is a fourier-related transform used to determine the frequency and phase content of local sections of a signal as it changes over time. In the discrete time case, the data to be transformed is broken into frames (which usually overlap each other to reduce the boundary effect). Each frame is Fourier transformed and the complex result is added to a matrix, which records the amplitude and phase for each point in time and frequency. This can be expressed as [24]:

$$
\operatorname{STPFT}\{x[n]\}(m, w)=\sum_{-\infty}^{\infty} x[n] w[n-m] e^{-j w n}
$$

with signal $\mathrm{x}[\mathrm{n}]$ and window $\mathrm{w}[\mathrm{n}]$. The window function is commonly a Hann window or Gaussian bell centered around zero. The magnitude squared of the STFT yields the spectrogram of the function [24]:

$$
\operatorname{spectrogram}\{\mathrm{x}(\mathrm{t})\}(\tau, \omega)=|X(\tau, w)|^{2}
$$

We can illustrate the different environmental contexts using spectrograms generated from the analysis of different audio background recordings. A spectrogram is a time-varying spectral representation which shows how the spectral density of a signal varies with time. The most common presentation format is a graph with two geometric dimensions: the horizontal axis represents time, the vertical axis is frequency; a third dimension indicating the amplitude of a particular frequency at a particular time is represented by the intensity or color of each point in the image. We use the Matlab source code provided by N. Seo [20] to analyze a couple of audio clips that either we collect or downloaded from the web. Four examples of the audio clips we analyze are shown in Fig 6. Fig 6(a) \& 6(b) show the spectrograms of different rooms, Fig 6(c) shows the audio clip of toilet flushing and Fig 6(d) shows the audio clip of telephone ringing. One can see that the spectrogram for different audio background is different and hence one can distinguish these different environments using certain audio features.

Our goal is to train a classifier with audio recordings of different environmental backgrounds so that we can identify the environment which an autistic child is in. Then, we can correlate the environments with the occurrences of stereotypical behaviors. To illustrate this, we asked a student who is sitting initially in an empty room to start tapping his 
foot when he hears the playing of an audio clip that mimics a party crowd. The top part of Fig 7 shows the output of our classifier that is trained to identify different actions performed by that student. The classifier sometimes misclassifies the action within an observation window (only 1 test instance) during the behavioral transition period to be "jumping" or "walking" but quickly determines that the student is performing foot tapping. It is easy to see from this example that if one observes many occurrences of such behaviors whenever a room becomes noisy, then we can add a rule to our knowledge based database to take intervention steps for that student whenever such an environment appears. We are currently in the process of fine tuning our audio-based classifier.

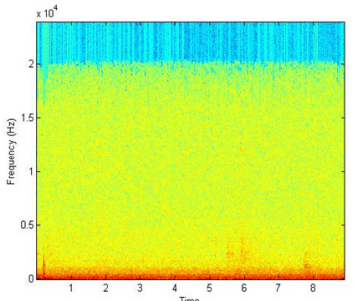

(a) Room1

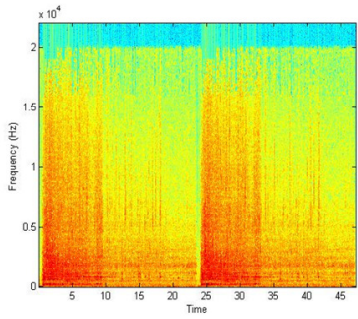

(c) Toilet Flushing

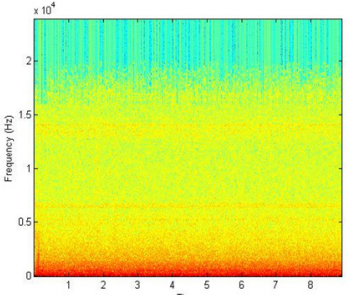

(b) Room4

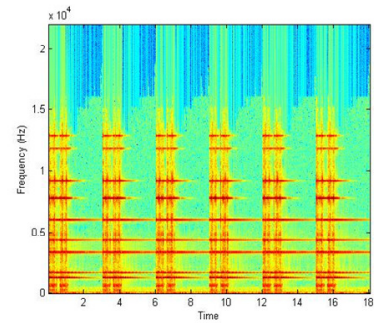

(d) Telephone Ringing
Fig 6 Spectrograms of different audio backgrounds

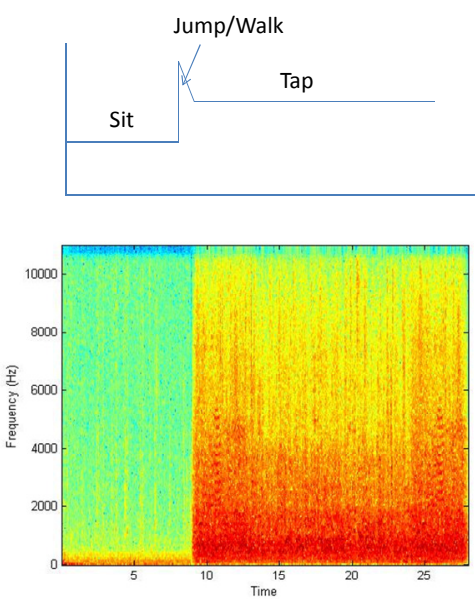

Fig 7 Correlating Environmental Factor with Stereotypical Behavior

In [25], the authors propose using an ambient sound fingerprint called Acoustic Background Spectrum (ABS) which can be easily computed to recognize the different rooms in campuses. Their indoor localization scheme based on ABS can yield an accuracy rate of $69 \%$. We evaluated this approach

using audio recordings of seven different environments we collected using Google or Samsung Nexus phones: on-going classes in classrooms, basketball games, birthday parties, empty rooms with air condition running, piano playing, phone conversations, shouting matches (most of which we obtained by playing video clips related to that topic from youtube). We have 820 -sec traces for each category. An example of the spectrogram for each category of audio signals we evaluated is shown in Fig 8. The ABS approach does not give us good accuracy since our sound environments also include some voice conversations, and music etc. Thus, we explore other approaches.

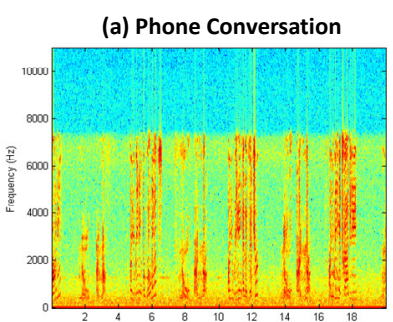

(c)Shouting Match

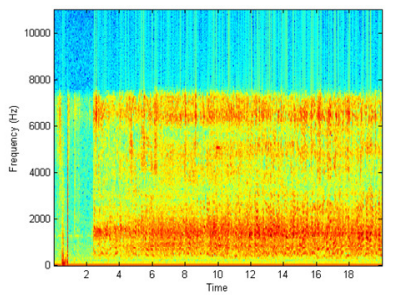

(e) BasketBallGame

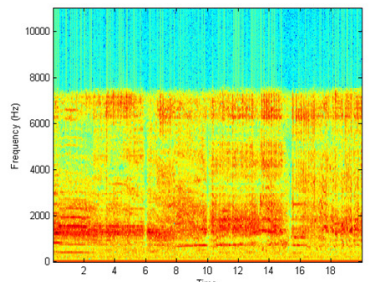

(b) Empty Room With AirCon on

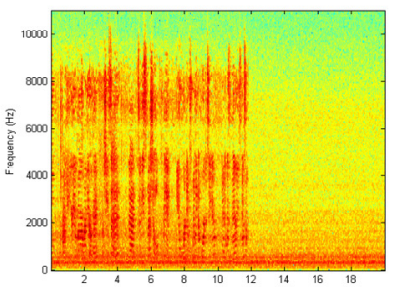

(d) Piano'Music

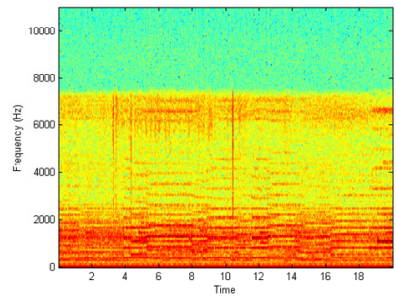

(f) BirthDayParty

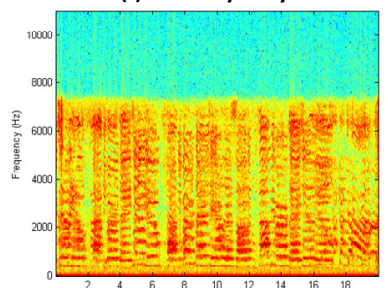

(g) Teaching in Classroom ${ }^{4}$

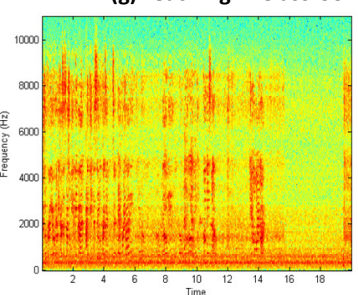

Fig 8 Spectrograms of Various Tested Audio Categories

In the end, we settled on an approach which is similar to the one described in [26] except that we use only 14 attributes: 12 Mel-frequency cepstral coefficients (MFCCs,) average zerocrossing rates, and energy (14 attributes). MFCCs are coefficients that collectively make up the mel-frequency cepstrum which is a representation of the short-term power spectrum of a sound based on a linear cosine transformation of a $\log$ power spectrum on a non-linear mel scale of frequency [27]. We extracted these 14 attributes from each set of traces we had using a tool called voicebox[28], trained these data 
using WEKA J.48 classifiers, and we can get an accuracy of $78.6 \%$ from the cross-validation experiment.

\section{Preliminary Prototype}

Next, we describe a preliminary prototype we have built on Android-based smartphones. Fig 9 shows the preliminary Android based application that we have developed for our Smartphone-based Autism Social Alert system. Our application currently can record accelerometer readings, audio background, and conduct Bluetooth discovery. The collected readings are sent via a $\mathrm{WiFi}$ connection to a server in our laboratory. Our server program is written in Java. It receives sensor data from the Android phone, inserts them into a mysql database which we created. Furthermore, the program can perform real-time analysis of the collected samples. It extracts relevant features from samples within an observation window (16 samples), create an instance that can be fed to our WEKA classifier to determine which behavioral action is currently being performed by a student. Our classifier currently only uses variances computed from the 3-axis accelerometer readings as features, and can currently recognize 5 behaviors, namely, sitting, walking, jumping, waving and foot tapping. We intend to add more behavioral recognitions e.g. body rocking in the near future.

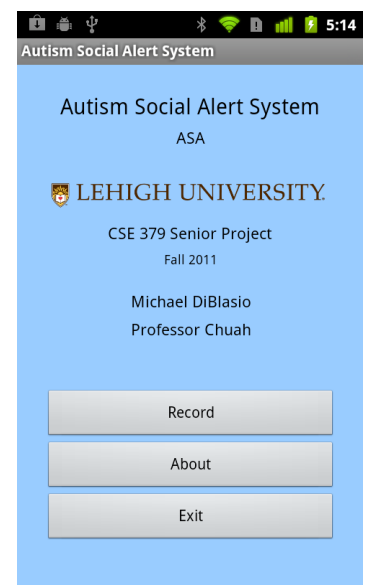

(a) Login Page

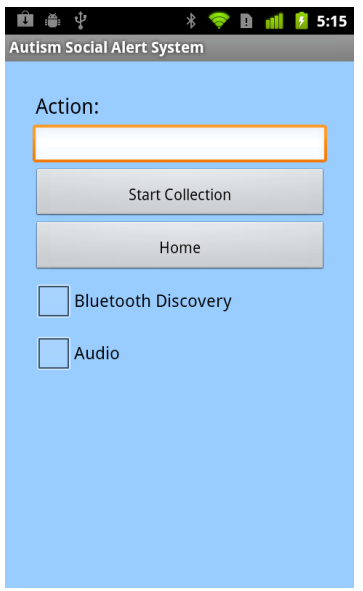

(b) Data Collection Page
Fig 9 Autsim Social Alert System

Since younger children most likely will not be granted access to smartphones, we also intend to use wearable sensors. In another senior design project, two female students designed a wearable sensor shown in Fig 10 which consists of accelerometer, skin temperature sensor, Bluetooth devices. We intend to enhance this design, and produce more working prototypes for our project if we cannot get cheaper off-theshelf wearable sensors that have similar features.
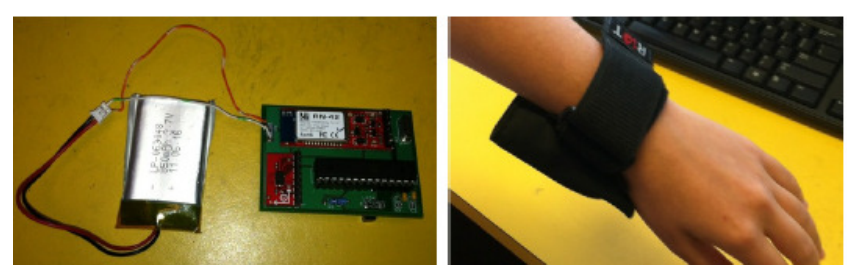

Fig 10 Wearable sensors built by students

\section{Preliminary Experimental Results}

We recruited 4 healthy students (Student1, Student2, Student3, and Student4) to be our volunteers in our preliminary experiments. For our experiments, we use an observation window of 16 samples (this is equivalent to $4 \mathrm{sec}$ worth of samples since our sampling rate is 4 samples/sec). We tried both independent and sliding window approaches (an overlap of 4 samples is used for the sliding window approach). In each of the experiments described below, the students are asked to perform only a single behavior in each collected trace.

The first set of experiments were conducted by Student1 using a HTC EVO device running Android 2.3.4. Student1 first collected 10 training datasets for each behavioral action (sitting, walking, hand waving, foot tapping, jumping). Except for hand-waving, the phone is placed in a pocket of that student's pants. For hand-waving, the phone is in that student's hand. We anticipate that in real scenario, wearable sensor will be attached to a student's wrist. We then train our WEKA J.48 classifier using his training datasets. Next, Student 1 collected additional datasets for the five actions which are then used for testing the accuracy of our WEKA classifier. The total accuracy rate for the test instances using independent and sliding window is $91 \%$ and $89 \%$ respectively. Since we want to evaluate real-time response time for behavior recognition, we opt for the sliding window approach for the rest of our experiments.

Next, we asked Student 2 to collect some test datasets when he performed similar behaviors using another device (a HTC Google device and refered to as Dev2). We then used Student's 1 training dataset and test with Student2's test datasets. The accuracy is still pretty good for three out of the five behaviors. The two behaviors with lower accuracy rates are the hand waving and foot tapping actions. Then, we asked Student 2 to collect more datasets which were then used to train our WEKA classifier to recognize Student 2's behaviors. Then, we test Student2's trained classifier with his own test datasets and the accuracy rates for all behaviors is now above $85 \%$.

To investigate if the training is needed for per user or per device type, we asked Student 2 to collect another dataset using a Samsung Google device (Dev3). Interestingly, we found that the classifier trained with HTC Google device can still give high accuracy rate $(>85 \%)$. To investigate if classifier trained with User2's behaviors can be used to recognize other users' behaviors using the same device, we asked Student 3 to collect datasets using both Google devices and Student 4 to collect his 
datasets using the Samsung Google device. The results are tabulated in Table1. One can see that as long as the users have similar walking paces, and perform similar actions, our classifier can yield high accuracy rate for identifying different types of behaviors. Student 4 walked faster than Student 2 and Student 3 and hence the accuracy rate drops to $76.5 \%$ but when Student4's walking traces are used as training set, the accuracy rises above $85 \%$.

\begin{tabular}{|l|r|r|r|r|r|}
\hline & \multicolumn{2}{|c|}{ Accuracy } & \multicolumn{2}{|c|}{ Accuracy } & \\
\hline & Dev2,User2 & Dev2, Use & Dev3 User2 & Dev3 User3 & Dev3 User4 \\
\hline Tapping & 0.869 & 0.9839 & 0.9515 & 0.947 & 0.9117 \\
\hline Waving & 1 & 1 & 0.899 & 0.9752 & 0.897 \\
\hline Walking & 0.909 & 0.8287 & 0.8763 & 0.8494 & 0.765 \\
\hline Jumping & 0.8513 & 0.9489 & 1 & 0.8426 & 0.9412 \\
\hline Sitting & 0.9577 & 0.9913 & 0.9779 & 0.9752 & 0.9759 \\
\hline
\end{tabular}

Table 1 Accuracy of Classifier with different devices \& users

We also collect some traces where Student2 performs different behaviors and our behavior recognition classifier was able to recognize different behaviors with high accuracy rates.

Our preliminary experiments reveal that most likely we need to train each user for each type of device he uses to achieve high accuracy rates. We may also have to train for different walking speeds of each monitored individual for we sometimes walk at different paces. Another observation is that a student's pants should not be loose such that the smartphone was tossed around inside the pocket during the data collection process. Otherwise, the smartphone needs to be clipped properly to a pocket in his pants.

Currently, we merely use the variances of the 3-axis accelerometer readings as features for our classifier. We intend to add in two additional features discussed in [13], namely (a) the correlation coefficients to capture the simultaneous motion in each axis, (b) FFT peaks and frequencies to capture differentiation between different intensities of the stereotypical behaviors to see if it can help to increase the accuracy of the classifier as we add more behaviors.

We intend to conduct more extensive experiments with healthy students to fine tune our system before we evaluate our system using volunteering students from Centennial School, a nearby secondary school for autism children [23] that Lehigh Education Department helps to run or some other special education schools for autistic students.

\section{CONCLUDING REMARKS}

In this paper, we have described a smartphone based autism social alert system which we built to monitor the autistic children's behaviors using embedded sensors in smartphones, and correlate with environmental related sensor readings that are collected simultaneously to identify possible environmental factors that may trigger stereotypical behaviors of such children. Once such an environmental factor is identified, intervention steps can be taken to prevent a student's emotional upsets or stereotypical behaviors. For example, one can present a positive response reminder in the form of an audio clip which contains the voice of a parent or teacher whom that student likes. Our system is still in the preliminary testing stage. When our system is more mature, we intend to deploy it at a nearby school for autistic children once we obtain an IRB approval. We are also in the process of enhancing the wearable sensor that we have developed for smaller children who may not have access to smartphones. Furthermore, we would also like to evaluate the impact of various error rates of wireless links on the detection accuracy for different SASA system deployments. In addition, we will add security and privacy design to our system to ensure that the system is robust against jamming attacks, and that the monitored traces can be stored in such a way that the users' privacy can be achieved.

\section{ACKNOWLEDGMENT}

We wish to thank J. Barberry, Dr. M. George from Centennial School, Dr. L. Bambara from the College of Education at Lehigh University for answering our questions at the early stage of this research. We also wish to thank S. Xin, Z. Qin for collecting traces using our SASA application.

\section{REFERENCES}

[1] J. Hoysniemi et al, "Shadow Boxer: a physically interactive fitness game", NordiCHI, 2004, 389-392.

[2] F. Butussi, L. Chittaro, "Smarter Phones for Healthier Lifestyles: An Adaptive Fitness Game", IEEE Pervasive Computing Vol 9 No 4, pp 51-57, 2010.

[3] A. Daley, "Can Exergaming Contribute to Improving Physical Activity Levels and Health Outcomes in Children?", Journal of American Academy of Pediatrics, Vol 124, No 2, pp 763-771,Aug, 2008.

[4] D.M. Papemy, J. R. starn, "Adolescant Pregnancy Prevention by Health Education Computer Games", Computer-Assisted Instruction of Knowledge and Attitudes", Pediatrics, Vol 83(5), 1989, 742-752

[5] R. Thomas, J. Cahill, L. Santili, "Using an Interactive Computer Game to Increase Skill and Self-Efficacy Regarding Safe Sex Negotiation: Filed Test Results", Health Education \& Behavior, Vol 24(1), 1997, 71-86.

[6] US CDC, "Prevalence of Autism Spectrum Disorders:Autism and Developmental Disabilities Monitoring Network, 14 sites, United States 2008”, March, 2012.

[7] P. Sturmey, "Video Technology and Persons with Autism and other Developmental Disabilities: An emerging technology for positive behavior support", Journal of Positive Behavior Interventions, Vol 5, 2003..

[8] D. A. Pyles, M. M. Riordan, J. S. Bailey, "The stereotypy analysis: An instrument for examining environmental variables associated with differential rates of stereotypic behavior", Research in Developmental Disabilities, Vol 18, pp 11-38,1997.

[9] J. A. Kentz, G. R. Hayes, T. L. Westeyn, T. Starner, G. D. Abowd, "Pervasive computing and autism: assisting 
caregivers of children with special needs", Pervasive Computing, Vol Jan-Mar, pp 28-35, 2007.

[10] T. Westeyn, K. Vadas, X. Bian, T. Starner, and G. D.Abowd, "Recognizing mimicked autistic selfstimulatory behaviors using HMMs," in Proceedings of ISW ,2005, pp. 164-169.

[11] M. Keally, G. Zhou, G. Xing, J. Wu, A. Pyles, "PBN: Towards Practical Activity Recognition Using Smartphone-Based Body Sensor Networks", Proceedings of ACM Sensys, Nov, 2011.

[12] M. Mahdaviani, T. Choudhury, "Fast and Scalable Training of Semi-Supervised CRFs with Application to Activity Recognition”, Proceedings of NPIS, Dec, 2007.

[13] F. Albinali, M. S. Goodwin, S. S. Intille, "Recognizing Stereotypical Motor Movements in the Laboratory and Classroom: A Case Study with Children on the Autism Spectrum”, Proceedings of ACM Ubicomp, 2009.

[14] B.A. Boyd et al, "Sensory Features and Repetitive Behaviors in Children with Autism", Autism Research, Vol 3 Issue 2, pp 78,87, April, 2010.

[15] M. H. Lewis, J. W. Bodfish, "Repetitive Behavior Disorders in Autism", Mental Retardation and Development Disabilities Research Review, Vol 4, pp 8089, 1998.

[16] C. H. Kennedy, K. A. Meyer, T. Knowles, S. Shukla, "Analyzing the multiple functions of stereotypical behaviors for students with autism: implications for assessment and treatment", Journal of Applied Behavioral Analysis, Winter, Vol 33(4), pp 559-571, 2000

[17] O.I. Lovaas, A. Litrownik, R. Mann, "Response latencies to auditory stimuli in autistic children engaged in selfstimulatory behavior", Behavior Research and Therapy, Vol 9, pp 39-49, 1971.

[18] R. F. Ristic, "Self-Injurious Behavior in People with Developmental Disabilities", Journal of Safe Management of Disruptive and Assaultive Behavior, pp 3-8, Sept, 2005.

[19] I. H. Witten, E. Frank, "Data Mining: Practical Machine Learning Tools and Techniques with Java Implementations", Morgan Kaufman, San Francisco, CA, 1999.

[20] N. Seo, "Short-Time Fourier Transform(Small)", note.sonots.com/SciSoftware/STFT.html.

[21] J. B. Allen, "Short term spectral analysis, synthesis, and modification by discrete Fourier transform", IEEE Transactions on Acoustics, Speech, Signal Processing, Vol 25, pp 235-238, June, 1977.

[22] T. Choudhury, S. Consolvo, B. Harrison, J. Hightower, A. LaMarca, L. LeGrand, A. Rahimi, A. Rea, "The Mobile Sensing Platform: An Embedded Activity Recognition System”, IEEE Pervasive Magazine, April, 2008.

[23] Lehigh College of Education Centennial School http://www.lehigh.edu/centennial

[24] Short-time $\quad$ Fourier $\quad$ Transform http://en.wikipedia.org/wiki/Short-time Fourier Transform

[25] S. P. Tarzia, P. A.Dinda, R.P. Dick, G. Memik, "Indoor Localization Without Infrastructure Using the Acoustic Background Spectrum", Proceedings of ACM MobiSys, June, 2011.

[26] K.H. Chang, D. Fisher, J. Canny, “AMMON: A Speech Analysis Library for Analyzing Affect, Stress, and Mental Health of Mobile Phones", $2^{\text {nd }}$ International Workshop on Sensing Applications on Mobile Phones (PhoneSense), 2011.

[27] Mel-frequency cepstrum, Wikipedia, http://en.wikipedia.org/wiki/Mel-frequency_cepstrum

[28] VOICEBOX: Speech Processing Toolbox for Matlab http://www.ee.ic.ac.uk/hp/staff/dmb/voicebox/voicebox.ht $\underline{\mathrm{ml}}$ 